neutral hydrogen model with uniform density and temperature is not valid in this region.

Using the lower limit of $1 \mathrm{kpc}$ to the distance of AP 2015, we can compute a lower limit to the mean electron density $\left\langle n_{e}\right\rangle$ in the direction of the pulsar. Adopting a dispersion measure of $14.2 \mathrm{pc} \mathrm{cm}^{-3}$ (ref. 9), we find $\left\langle n_{e}\right\rangle$ $<0.015 \mathrm{~cm}^{-3}$. This is significantly smaller than usually assumed for the interstellar medium.

\title{
P. Encrenaz
} M. Gútin

Observatoire de Meudon,

France.

Received April 20, 1970.

${ }^{1}$ De Jager, G., Lyne, A. G., Pointon, L., and Ponsonby, J. E. B., Nature, 220, $128(1968)$.

${ }^{2}$ Guélin, M., Guibert, J., Fuchtmeier, W., and Weliachew, L., Nature, 221, 249 (1969).

${ }^{3}$ Gordon, C. P., Gordon, K. J., and Shalloway, A. M., Nature 222, 129 (1969).

'Hjellming, R. M., Gordon, C. P., and Gordon, K. J., Astron. Astrophys., 2, $202(1969)$.

'Manchester, R. N., Murray, J. D., and Radhakrishnan, V., Astrophys. Lett., 4, 229 (1969).

6 Muller, C. A., and Westerhout, G., Bull. Astron. Inst. Netherlands, 13, 151 (1957).

' Clark, B. G., Astrophys. J., 142, 1398 (1965),

schmidt, M., in Galaetic Structure (Stars and Stellar Systems, 5) (edit. by Blaauw, A., and Schmidt, M.) (Univ. Chicago Press, 1965).

- Craft, Jun., H. D., Sutton, J. M., Comella, J. M., and Lovelace, T. V. E., Nature,219, 1237 (1968).

\section{Displacement of the Clouds of Venus}

THE apparent four day rotation of the cytherean atmosphere was first pointed out by Boyer (private communication) in 1957 and the retrograde sense of the rotation was discovered by Boyer and Camichel ${ }^{1}$ from the cloud markings often visible on ultraviolet photographs. The presence of this rotation has been reported several times

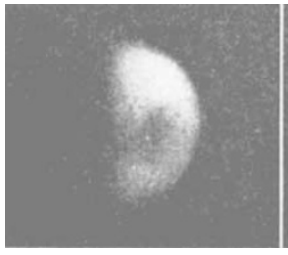

0422

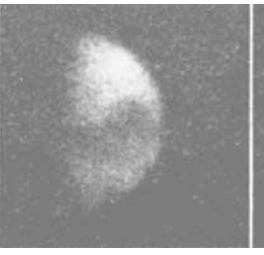

0523

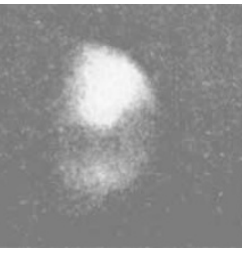

0720
Fig. 1. The short term evolution of a cloud pattern.

since (refs. 2-8 and unpublished work of Kuiper et al.). The periodicity shows itself in two ways: $(a)$ any particular formation, if observed over a period of hours, demonstrates a retrograde rotation of period approximately four days, although the periods obtained in this way scatter about a mean value; (b) large scalo markings with easily recognizable shapes reappear at intervals of four days or multiples of this value, and the appearance of particular formations can be predicted for long periods in advance. Fig. 1 shows the short term evolution for a type of cloud pattern often obscrved.

We selected the best photographs of Venus taken in ultraviolet light available at the IAU Planetary Data Centre at Meudon Observatory, and measured the longitudes and latitudes of the well-defined formations (called $Y, \Psi^{*}$ and $H$ by Boyer) on 300 plates taken during the years 1966-69. We dotermined the movement of the clouds with time in each time sequence. Then the longitudes of the clouds were averaged in one-hour intervals to get the mean displaccment of the individual clouds with time. The results are shown in Fig. 2 indicating a rotation period of $4 \cdot 0 \pm 0.5$ days retrograde for the upper

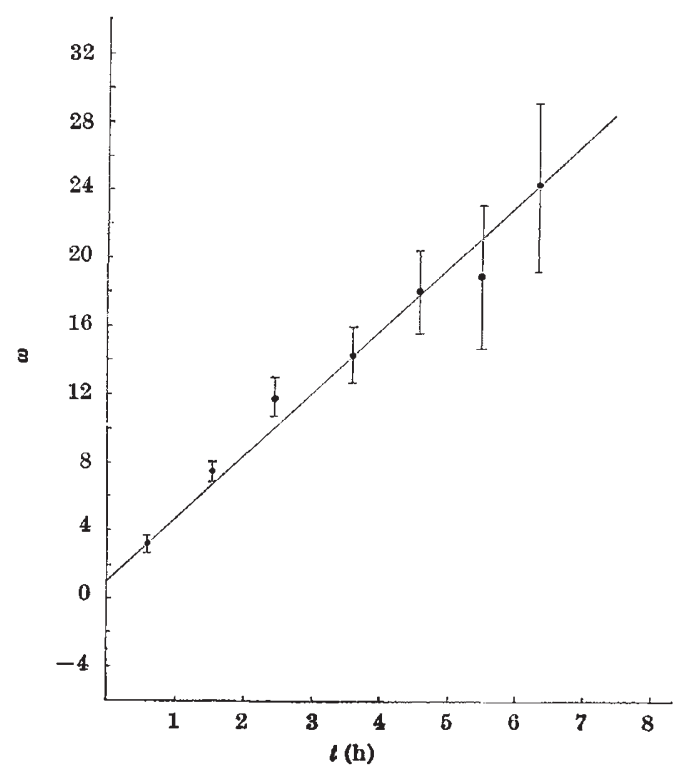

Fig. 2. The displacement of individual clouds in series of plates averaged over intervals of one hour as a function of time. The line corresponds indicate the Gaussian probable error of the measurements.
ine bars

atmosphere of Venus. Omitting the errors, we derived from the dispersion of the individual clouds an upper limit of about $740 \mathrm{~km} \mathrm{~h}^{-1}$ for the wind velocity, if we suppose that the clouds are at an altitude of about $95 \mathrm{~km}$ from the radar surface $(R=6,052 \mathrm{~km})$ of Venus (Kuiper, unpublished). The corresponding minimum velocity would be about $320 \mathrm{~km} \mathrm{~h}^{-1}$.

The reality of this bulk rotation of the upper atmosphere has been demonstrated independently by Guinot? by Doppler techniques and a Fabry-Pérot interferometer in the wavelength range $5500-5700 \AA$. The agreement with the photographically derived period is excellent, the present best estimate reported by Guinot and Feissel ${ }^{8}$ being $4 \cdot 3 \pm 0 \cdot 4$ days retrograde, provided the axis of rotation is perpendicular to the orbital plane. As these recent observations on which this figure is based were made at a variety of elongations over a period of years, it seems reliable. The agreement between these two different observational methods is strong evidence in favour of the rapid bulk motion of the cytherean upper atmosphere. The difference between the radar period of 243 days retrograde and the ultraviolet period of four days retrograde possibly indicates a complex planetary atmospheric circulation, the nature of which is obscure.

JUKKA NIKANDER

Aarne Karjalainen Observatory,

University of Oulu,

Oulu, Finland.

Observatoire du Pic du Midi,

Ch. Boyer

Bagnères de Bigorre,

France.

Received April 8, 1970.

${ }^{1}$ Boyer, C., and Camichel, H., Ann. Astrophys., 24, 531 (1961).

${ }^{2}$ Boyer, C., L'Astronomie, 79, 223 (1965)

Boyer, C., and Camichel, H., CR Acad. Sci., 260, 809 (1965).

- Boyer, C., and Guérin, P., CR Acad. Sci., 263, 253 (1966).

'Dollfus, A., in The Atmospheres of Venus and Mars (edit. by Brandt, I., and McElroy, M.) (Gordon and Breach, 1968).

- Smith, B. A., Seience, 158, 114 (1967).

7 Guinot, B, CR Acad. Sci, 260, 431 (1965).

'Guinot, B., and Feissel, M., J. des Observateurs, 51, 13 (1968). 